\title{
Beobachtungen von Jupiterstrabanten
}

am Utrechter Refractor (Oeffnung $26 \mathrm{~cm}$, Brennweite $319 \mathrm{~cm}$, Vergrösserung 248).

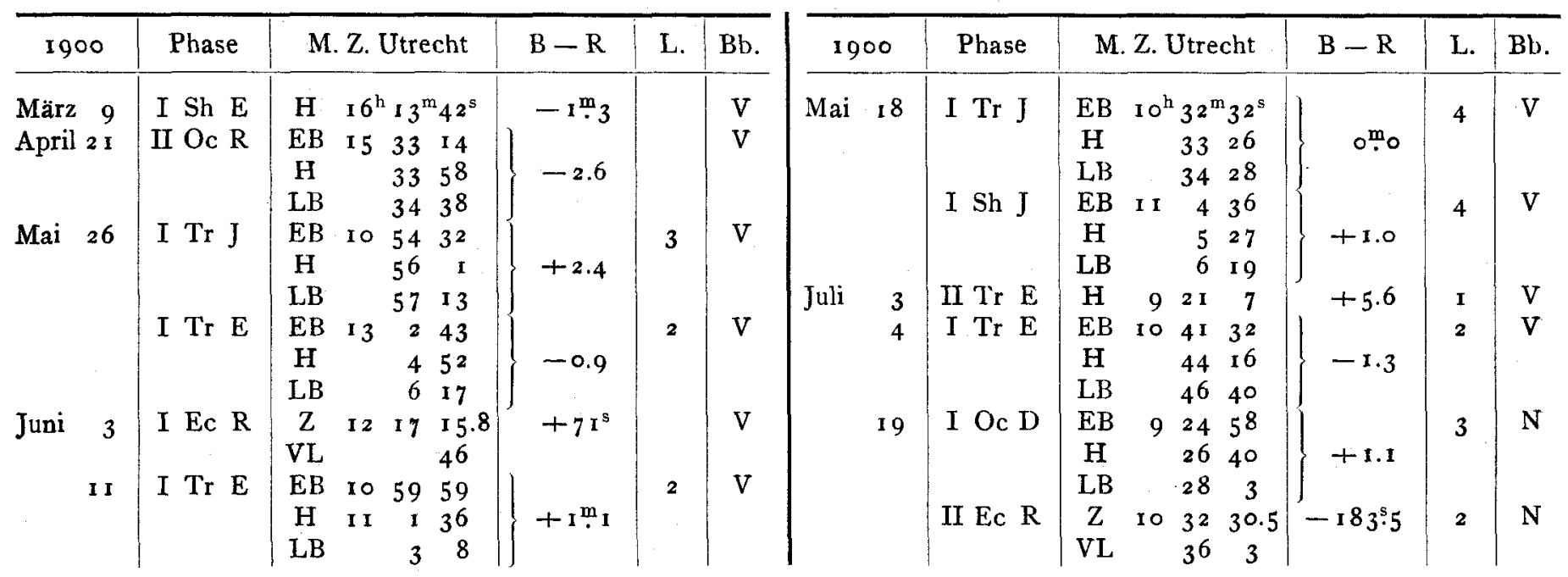

Bemerkungen.

Juni I 8, I Tr J. Gut. Bilder sehr ruhig. - Juli 3, II Tr E. Unsicher. - Juli I9, I Oc D. Ziemlich gut. Juli I 9, II Ec R. Leidlich. Planet niedrig. Nebel.

$\mathrm{EB}=$ erste Berührung; $\mathrm{H}=$ Halbirung; $\mathrm{LB}=$ letzte Berührung; $\mathrm{Z}=$ Trabant zuerst gesehen; $\mathrm{VL}=$ Trabant hat das volle Licht.

Utrecht, 1901 März 8.
Die Spalte $B-R$ enthält den Unterschied Beobachtung minus Angabe des Nautical Almanac. Für die östliche Länge von Utrecht haben wir $20^{\mathrm{m}} 33^{\text {s.o }}$ angenommen. In der mit L. bezeichneten Spalte ist der Luftzustand angegeben; 1, 2, 3 und 4 bedeuten der Reihe nach schlecht, ziemlich gut, gut und ausgezeichnet.

\section{A. A. Nijland, S. L. Veenstra.}

\section{Observations of Comet $1901 \mathrm{I}$}

made at the Royal Observatory Cape of Good Hope, by Mr. R. T. A. Innes.

(Communicated by Sir David Gill, H. M. Astronomer).

\begin{tabular}{|c|c|c|c|c|c|c|c|c|c|c|c|c|c|c|c|c|c|c|c|c|c|}
\hline \multicolumn{2}{|c|}{$190 \mathrm{I}$} & \multicolumn{3}{|c|}{ Greenw.M.T. } & \multicolumn{2}{|c|}{$\Delta \alpha$} & \multicolumn{2}{|c|}{$\Delta \delta$} & \multirow{2}{*}{$\frac{\text { Cp. }}{7.7}$} & \multicolumn{3}{|c|}{$\alpha$ app. } & \multirow{2}{*}{$\frac{\log p \cdot \Delta}{9.682}$} & \multicolumn{3}{|c|}{$\delta$ app. } & \multirow{2}{*}{$\frac{\log p \cdot \Delta}{0.688_{\mathrm{n}}}$} & \multicolumn{3}{|c|}{ Red. ad l. app. } & \multirow{2}{*}{$\frac{*}{\mathbf{I}}$} \\
\hline 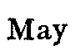 & 3 & $5^{h}$ & & ${ }^{m} 43^{s}$ & $+0^{n}$ & $\mathrm{~m}_{39} \cdot \mathrm{s} 5$ & + & $4^{\prime}$ I $44^{\prime \prime}$ & & & $40^{r}$ & ${ }^{n} 3^{2} \cdot 39$ & & $-0^{\circ}$ & ${ }^{\circ} 3^{\prime}$ & 18.6 & & +0.52 & - & $3: 7$ & \\
\hline & 4 & & 15 & & -3 & 8.60 & +1 & 351.6 & 6.6 & 3 & 54 & 24.23 & 9.682 & -0 & I 8 & 27.9 & $0.688_{n}$ & +0.55 & - & 4.6 & 2 \\
\hline & 5 & 4 & 57 & 46 & +3 & 4.16 & + & $\begin{array}{ll}3 & 33.7\end{array}$ & 4.4 & 4 & 7 & 8.97 & $9.67 \mathrm{I}$ & -0 & $\mathbf{I}$ & 32.4 & $0.689 \mathrm{n}$ & +0.56 & - & 5.0 & 3. \\
\hline & 5 & 5 & 29 & 24 & -3 & 31.76 & $-I$ & 328.3 & 4.4 & 4 & 7 & 25.23 & 9.683 & -0 & I & 8.5 & $0.689 \mathrm{n}$ & $+0.5^{8}$ & - & $5 \cdot 3$ & 4 \\
\hline & 6 & 4 & 49 & 45 & +8 & 10.38 & + & $5 \quad 57.5$ & I. I & 4 & 19 & $7 \cdot 37$ & $9.66 \mathrm{I}$ & +0 & 18 & 17.4 & $0.689 \mathrm{n}$ & $+0.5^{8}$ & - & 5.2 & 4 \\
\hline & 6 & & » & & -10 & I 5.53 & + & 616.1 & r. $\mathrm{I}$ & 4 & 19 & 7.04 & $»$ & +0 & 18 & 21.5 & $\gg$ & $+0.6 \mathrm{I}$ & - & 6.2 & 5 \\
\hline & 6 & 5 & 5 & 48 & +1 & 1.62 & + & I 57.7 & 5.4 & 4 & 19 & $\mathrm{I}_{4.9}$ I & $9.67 \mathrm{I}$ & +0 & 18 & 34.8 & $0.689 n$ & +0.59 & - & $5 \cdot 6$ & 6 \\
\hline & 6 & 5 & 15 & 18 & -1 & $3^{8.57}$ & - & $8 \quad 35.8$ & 5.5 & 4 & 19 & $19.2 \mathrm{I}$ & 9.675 & +0 & 18 & 35.8 & $0.689 \mathrm{n}$ & +0.60 & - & $5 \cdot 7$ & 7 \\
\hline & 7 & 5 & $\mathbf{I}$ & 15 & $-I$ & 43.89 & - & $7 \quad 30.8$ & 8.8 & 4 & 30 & 24.24 & 9.663 & +0 & 40 & 15.0 & $0.687 \mathrm{n}$ & +0.62 & - & 6.2 & 8 \\
\hline & I I & 4 & $5^{\circ}$ & 17 & -0 & 58.82 & - & 129.0 & 6.6 & 5 & 7 & 38.75 & $9.63^{8}$ & +2 & I 3 & 45.0 & $0.699 \mathrm{n}$ & +0.71 & - & 7.6 & 9 \\
\hline & 11 & 5 & 7 & 28 & -0 & 50.01 & + & 9 I.I & $5 \cdot 5$ & 5 & 7 & 42 & 9.653 & +2 & I 4 & & $0.698_{n}$ & $+0.7 \mathrm{I}$ & - & $7 \cdot 6$ & 10 \\
\hline & I 2 & 4 & 49 & 56 & -0 & I 9.49 & $-I$ & 242.3 & 2.2 & 5 & 15 & $22.7 \mathrm{I}$ & 9.633 & +2 & $3^{6}$ & 50.6 & $0.702 \mathrm{n}$ & +0.74 & - & 7.8 & I I \\
\hline & 12 & 5 & 37 & 58 & +1 & 35.92 & + & $8 \quad 12.2$ & 6.6 & 5 & $r_{5}$ & 38.02 & 9.669 & +2 & 37 & $39 \cdot 3$ & $0.697 \mathrm{n}$ & +0.73 & - & $7 \cdot 7$ & 12 \\
\hline & 13 & 4 & 59 & 28 & +0 & 58.94 & - & ○ 48.3 & 6.6 & 5 & 22 & 38.72 & 9.640 & +2 & 59 & 39.6 & $0.7 \circ 3 n$ & +0.75 & $\rightarrow$ & 8.0 & 13 \\
\hline & 15 & 4 & 45 & 49 & -0 & 37.84 & - & 052.1 & 10.10 & 5 & 35 & 36.67 & $9.62 \mathrm{I}$ & +3 & 42 & $36: 7$ & $0.709 \mathrm{n}$ & +0.80 & & 8.5 & I 4 \\
\hline & 16 & 4 & 37 & 44 & -0 & 48.14 & - & 042.7 & 10.10 & 5 & $4 \mathrm{I}$ & 29.04 & $9.6 \circ 9$ & +4 & 2 & 57.4 & $0.7 \times 2 \mathrm{n}$ & +0.81 & - & 8.7 & I 5 \\
\hline & 17 & 4 & $5 I$ & 25 & +2 & $5 \cdot 39$ & & - 39.9 & 10.10 & 5 & 47 & 4.81 & 9.623 & +4 & 24 & I I .4 & $0.712 \mathrm{n}$ & +0.82 & - & 8.8 & 16 \\
\hline & 18 & 4 & 52 & 17 & $+\mathbf{r}$ & 53.88 & & I 50.8 & 6.6 & 5 & $5^{2}$ & 18.46 & 9.623 & +4 & 41 & 48.6 & 0.7 I $4 n$ & +0.84 & - & 9.0 & 17 \\
\hline & 19 & 4 & 50 & 53 & +0 & 57.99 & & $\begin{array}{ll}5 & 27.9\end{array}$ & 10.10 & 5 & 57 & 13.79 & $9.62 \mathrm{I}$ & +4 & 59 & $55 \cdot 5$ & $0.716_{n}$ & +0.85 & - & 9.2 & 18 \\
\hline & 20 & 5 & 7 & 53 & +2 & I 3.38 & & 753.6 & 10.10 & 6 & $\mathbf{I}$ & 55.69 & 9.640 & +5 & 17 & 28.7 & $0.714 \mathrm{n}$ & +0.86 & - & 9.2 & 19, \\
\hline
\end{tabular}




\begin{tabular}{|c|c|c|c|c|c|c|c|c|c|c|c|c|c|c|c|c|}
\hline \multicolumn{2}{|c|}{1901} & Greenw.M.T & & \multicolumn{2}{|r|}{$\Delta \delta$} & Cp. & \multicolumn{3}{|c|}{$\alpha$ app. } & $\log p . \Delta$ & \multicolumn{2}{|c|}{$\delta$ app. } & $\log p \cdot \Delta$ & \multicolumn{2}{|c|}{ Red. ad l. app. } & * \\
\hline & 21 & $5^{\mathrm{h}} \times 7^{\mathrm{m}} 54^{\mathrm{s}}$ & $-0^{m} 3^{8.993}$ & + & $7^{\prime}$ I 4.5 & 12.12 & $6^{\mathrm{h}}$ & $6^{\mathrm{m}}$ & n & 9.647 & $+5^{\circ} 34^{\prime}$ & & 0.713 & +0.87 & $-9 \cdot 3$ & 20 \\
\hline & 22 & $4 \quad 4^{6} \quad 4^{2}$ & to 48.87 & + & 152.4 & 10.10 & 6 & 10 & & 9.614 & +549 & 48.9 & $0.722 \mathrm{n}$ & +0.89 & -9.4 & $2 \mathrm{x}$ \\
\hline & 23 & 5226 & $+04 \mathrm{I} \cdot 3 \mathrm{I}$ & - & 244.1 & I $5: 15$ & 6 & 14 & & 9.632 & +6 & I I.O & $0.719 \mathrm{n}$ & +0.91 & -9.4 & 22 \\
\hline & 24 & $\begin{array}{lll}5 & 5 & 17\end{array}$ & 2.81 & 一 & $0 \quad 27.9$ & 10.10 & 6 & 18 & & $9.63^{6}$ & +619 & 7.0 & $2 O_{n}$ & +0.92 & -9.6 & 23 \\
\hline & 27 & $4 \quad 5^{6} \quad 3^{2}$ & to 24.03 & + & - 32.5 & 12.12 & 6 & 28 & . & 9.629 & +659 & & $24 n$ & +0.94 & -9.6 & 24 \\
\hline & 28 & $5 \circ 18$ & $\begin{array}{ll}-1 & 3.39\end{array}$ & - & I 55.3 & I. I & 6 & 31 & 9 & 9.634 & +7 I I & 56.0 & $0.723 n$ & +0.96 & -9.7 & 25 \\
\hline & 29 & $4 \quad 58 \quad 26$ & to 48.14 & + & 233.6 & 4.4 & 6 & 34 & & 9.633 & +723 & 32.1 & $24 n$ & +0.96 & -9.7 & 26 \\
\hline & 30 & $\begin{array}{lll}4 & 48 & 29\end{array}$ & $-05^{1.38}$ & - & $0 \quad 11.7$ & 15.15 & 6 & 37 & & 9.623 & +734 & 38.6 & $0.727 \mathrm{n}$ & +0.97 & -9.8 & 27 \\
\hline e & 2 & $4 \quad 5520$ & -1 6.19 & + & 430.0 & 10.10 & 6 & 45 & $\mathbf{I}$ & 9.635 & +85 & 20.2 & $0.727 n$ & +0.99 & -10.0 & 28 \\
\hline & 4 & 5730 & +046.60 & - & 310.9 & I 5.15 & 6 & 50 & 59. & $9.65^{\circ}$ & +823 & 39.8 & $0.723 n$ & +1.01 & -9.9 & 29 \\
\hline & 8 & $\begin{array}{lll}4 & 49 & 30\end{array}$ & to $3 \pm$ & + & - 34.0 & -.11 & 7 & 0 & 8 & - & +855 & 41.7 & $.729 n$ & +1 & -9.9 & 30 \\
\hline & 8 & $\begin{array}{lll}5 & 9 & 3\end{array}$ & - I 11.45 & + & 346.7 & 12.12 & 7 & $\circ$ & 8.68 & 9.657 & +855 & 50.0 & $0.72 I_{\mathrm{n}}$ & +1.04 & -10.0 & $3^{r}$ \\
\hline & 9 & $\begin{array}{lll}4 & 53 & 34\end{array}$ & -0 24.15 & - & 320.2 & 13.12 & 7 & 2 & 15.29 & 9.646 & +9 & 53.7 & $0.727 \mathrm{n}$ & +1.04 & -9.9 & $3^{2}$ \\
\hline & 10 & $5 \bigcirc 41$ & to 12.78 & - & $4 \quad 4.0$ & 12.12 & 7 & 4 & 20.39 & 9.655 & +9 & 50.1 & $0.724 n$ & +1.05 & -9.9 & 33 \\
\hline & I 2 & 23 & +124.66 & - & 014.0 & 15.15 & 7 & 8 & 2 I.II & 9.658 & +922 & 44.2 & $0.723 n$ & +1.06 & -9.9 & 34 \\
\hline & 13 & 45329 & -o 53.97 & - & I 48.7 & 10.10 & 7 & ro & 16.39 & 9.653 & +928 & $4 \mathrm{I} .2$ & $0.726 \mathrm{n}$ & +1.07 & -10.0 & 35 \\
\hline & 14 & $\begin{array}{lll}457 & 8\end{array}$ & to 43.78 & + & $2 \quad 26.8$ & 16.16 & 7 & 12 & 9.68 & 9.658 & +934 & $3^{2.5}$ & $0.724 n$ & +1.07 & -9.9 & $3^{6}$ \\
\hline
\end{tabular}

Mean places of the comparison stars.

\begin{tabular}{|c|c|c|c|c|c|c|c|c|c|c|c|c|}
\hline$\approx$ & \multicolumn{3}{|c|}{$\alpha 1901.0$} & \multicolumn{2}{|c|}{$\delta 1901.0$} & Authority & $*$ & \multicolumn{2}{|c|}{$\alpha$ I 901.0} & \multicolumn{2}{|c|}{$\delta 1901.0$} & Authority \\
\hline I & & 39 & $52 \cdot 72$ & $-0^{\circ} 3^{\prime}$ & $29 " 3$ & Cape $_{90}$ & 19 & & $59^{m} 41^{s} \cdot 45$ & $+5^{\circ} 25^{\prime}$ & 31.5 & $1 / 2($ AG. $+10 y r)$. \\
\hline 2 & 3 & 57 & 32.28 & $-03^{2}$ & 14.9 & $1 / 3\left(\right.$ 10yr. $\left.+\operatorname{Rad}_{3}+\mathrm{Cape}_{80}\right)$ & 20 & & 70 & +527 & & BD. $+5^{\circ} \times 1 \times 30$ \\
\hline & & & & & & with p. m. from $\operatorname{Rad}_{3}$ & 21 & & $937 \cdot 3^{2}$ & +548 & 5.9 & AG. \\
\hline 3 & 4 & 4 & 4.25 & - 05 & I.I & $1 / 3($ Paris +2 AG. $)$ & 22 & 61 & 346.01 & +68 & $4 \cdot 5$ & $A G$. \\
\hline 4 & & 10 & 56.41 & to 12 & $25 \cdot r$ & $\mathrm{AG}$ & 23 & 62 & $20 \quad 17.97$ & +620 & 24.5 & $A G$. \\
\hline 5 & 4 & 29 & 21.96 & +012 & I 1.6 & AG. (p. m. o.o, - - ". 1 I) & 24 & 62 & 289 & +659 & & BD. $+7 \circ 134 \mathrm{I}$ \\
\hline 6 & 4 & 18 & 12.70 & $+0 \quad 16$ & 42.7 & AG. & 25 & 63 & 3251.22 & +714 & 1.0 & AG. \\
\hline 7 & 4 & 20 & 57.18 & +027 & 19.7 & AG. & 26 & & $34 \quad 1.95$ & +721 & 8.2 & $A G$. \\
\hline 8 & 4 & $3^{2}$ & $7 \cdot 5 \mathrm{I}$ & +047 & 52.0 & $1 / 2(\mathrm{AG} .+9 \mathrm{yr})$. & 27 & 63 & $38 \quad 36.11$ & +735 & o. I & AG. $\left(\mathrm{An} . \mathrm{I}^{\mathrm{m}}\right.$ comes $5^{\prime \prime} \mathrm{nf}$.) \\
\hline 9 & 5 & 8 & 36.86 & +215 & 2 I.6 & $1 / 2$ (AG. + Yarn.) & 28 & 64 & $47 \quad 2.6 \mathrm{I}$ & $+8 I$ & 0.2 & $A G$ \\
\hline 10 & 5 & 8 & 31 & +25 & & BD. +2.890 & 29 & 65 & 5011.60 & +827 & 0.6 & AG. \\
\hline I I & 5 & I 5 & $41 \cdot 4^{6}$ & +249 & 40.7 & AG. & 30 & 7 & 04.41 & +855 & 7.6 & $A G$. \\
\hline 12 & 5 & I 4 & $1 \cdot 37$ & +229 & 34.8 & AG. & $3 I$ & 7 & I $\quad 19.09$ & $+85^{2}$ & I $3 \cdot 3$ & AG. (p. m. o. $00,-0.1$ ) \\
\hline 13 & 5 & $2 \mathbf{I}$ & 39.03 & $+3 \circ$ & 35.9 & AG. (p.m. $-0.0009,-0.004)$ & $3^{2}$ & 7 & 238.40 & +96 & 23.8 & AG. \\
\hline 14 & 5 & $3^{6}$ & 13.71 & +343 & $37 \cdot 3$ & AG. & 33 & & $4 \quad 6.56$ & +9 I 4 & 4.0 & $A G$. \\
\hline $\mathbf{I} 5$ & 5 & 42 & 16.37 & +43 & 48.8 & AG. (p.m. ..., -о"॰3) & 34 & 7 & $6 \quad 55 \cdot 39$ & +923 & 8. 1 & $\mathrm{AG}$. \\
\hline 16 & 5 & 44 & $5^{8.60}$ & +423 & 40.3 & $1 / 2(\mathrm{AG} .+10 \mathrm{yr})$. & 35 & 7 & $11 \quad 9.29$ & +930 & 39.9 & AG. \\
\hline 17 & & 50 & 23.74 & +443 & 48.4 & $\mathrm{AG}$. & $3^{6}$ & 7 & I I 24.83 & $+93^{2}$ & 5.6 & $A G$. \\
\hline 18 & & 56 & I 4.95 & +454 & 36.8 & AG. & & & & & & \\
\hline
\end{tabular}

Notes.

The observation of $3^{\mathrm{d}}$ May $190 \mathrm{r}$ was made with the I 8 inch Mc Clean refractor, the others with the 7 inch refractor - all with the Repsold filar micrometer. Differential refraction has been applied to both elements.

The column »Red. ad l. app. « applies to the comparison stars and not to the comet.

May 5. The observations on this date were difficult as the comparison stars were very faint. - May 7. Diameter of nucleus $=16$ " by one double distance. - May I I. Nucleus much small and fairly stellar. - May I 3. The fainter branch of the main tail passed exactly over the central star of Orion's belt. -- May 15. Comet is double, angle $10 \% 6$, dist. 0.8 or 1.0 , difference of magnitudes 1.0 . There is an extension of coma in the direction $10: 6$ as well. Tail extends from $5^{\mathrm{h}} 3^{6^{\mathrm{m}}}+3^{\mathrm{o}} 4^{\circ}$ to $6^{\mathrm{h}} 2^{\mathrm{ni}}+4^{\circ} 27^{\circ}$ in 7 in. and to $6^{\mathrm{h}} 15^{\mathrm{m}}+4^{\circ} 30^{\prime}$ in the 2 in. finder. - May 18 . Nucleus quite stellar (in other words the elongation or separation of the $15^{\text {th }}$ had disappeared). - May 2 I. Comet diffused and difficult to bisect. - May 23. Hazy. - May 24. Passing clouds. - May 27. Tail 50' long in 7 in. May 28-29. Stopped by cloud. - May 30. Tail 6' long. June 2. Nucleus $=99^{\mathrm{m}} 5 \mathrm{star}$, coma about $60^{\prime \prime}$ diameter, no tail noticed, passing clouds. - June 4. Nucleus $=10^{\mathrm{m}}{ }_{5} \mathrm{star}$, 
coma about $30^{\prime \prime}$ diameter, tail perhaps $2^{\circ}$ long in finder. faint. The tail can still be traced over several degrees Comet near a 9.0 mag. star. - June 8. Long narrow tail in a low power field. Nucleus ill defined. - June ro. still visible. - June 9. Illumination of wires bad and comet Nucleus not steadily held. Zenith distance $72^{\circ}-82^{\circ}$.

Royal Observatory, Cape of Good Hope, rgor June 27.

David Gill, H. M. Astronomer.

Elemente und Aufsuchungs-Ephemeride des Encke'schen Cometen.

Epoche und Osculation 190 I Juli 8.0 M. Z. Berlin.

$$
\left.\begin{array}{rlrl}
M & =339^{\circ} 20^{\circ} 39^{\prime \prime} \cdot 38 \\
\pi & =15847 & 57.37 \\
\delta & =334 & 48 & 58.06 \\
i & =12 & 53 & 38.46
\end{array}\right\} \text { M. Aequ. 1901.0 } \quad \begin{aligned}
\varphi & =57^{\circ} 46^{\circ} 44^{\prime \prime 82}-2.394 \tau \\
& =1073^{\prime \prime} 87571+0.069299 \tau
\end{aligned}
$$

\begin{tabular}{|c|c|c|c|c|c|c|c|}
\hline \multicolumn{2}{|c|}{1901} & \multicolumn{2}{|c|}{$\alpha$ app. } & $\delta$ app. & $\log r$ & $\log \Delta$ & Ab.-Z. \\
\hline ug. & 5 & $5^{\mathrm{h}} 57^{\mathrm{m}}$ & $8^{s}$ & $+31^{\circ} 42^{\prime} \cdot 3$ & $9.99 \times 6$ & 0.1534 & I $1^{m} 5^{\mathrm{s}}$ \\
\hline & 6 & 633 & $3^{6}$ & 314 I.I & 9841 & 1494 & 43 \\
\hline & 7 & IO I & 12 & $31 \quad 38.4$ & 9765 & I 454 & 37 \\
\hline & 8 & 165 & 55 & $31 \quad 34.4$ & 9687 & 1416 & 31 \\
\hline & 9 & 234 & 44 & 3129.0 & 9607 & I 379 & 25 \\
\hline & 10 & 30.4 & 40 & 3122.2 & 9525 & 1343 & 19 \\
\hline & 11 & 374 & $4^{2}$ & $31 \quad 13.7$ & $944 I$ & $13 \circ 7$ & 14 \\
\hline & I 2 & 445 & $5^{2}$ & $3 \cdot 5$ & 9354 & I 273 & 9 \\
\hline & 13 & $5^{2}$ & 7 & 3051.5 & 9265 & 1241 & I I 4 \\
\hline & I 4 & 6592 & 29 & $\begin{array}{ll}3 \circ & 37.8\end{array}$ & 9173 & I 2 I 0 & $10^{\circ} 59$ \\
\hline & 15 & 765 & $5^{6}$ & +3022.2 & 9.9080 & 01181 & 10 54 \\
\hline
\end{tabular}

\begin{tabular}{|c|c|c|c|c|c|c|}
\hline \multicolumn{2}{|c|}{ IgOI } & $\alpha$ app. & $\delta$ app. & $\log r$ & $\log \Delta$ & Ab.-Z. \\
\hline ug. & I 5 & $7^{\mathrm{h}} \quad 6^{\mathrm{m}} 5^{6^{\mathrm{s}}}$ & $+30^{\circ} 22 ! 2$ & 9.9080 & $0.118 \mathrm{I}$ & $10^{\mathrm{m}} 54^{\mathrm{s}}$ \\
\hline & 16 & 1429 & $30 \quad 4.7$ & 8984 & I I 53 & 50 \\
\hline & I 7 & 227 & 2945.1 & 8885 & I I 27 & 46 \\
\hline & I 8 & 2949 & $29 \quad 23.5$ & 8783 & 1103 & 42 \\
\hline & 19 & 3736 & $28 \quad 59.6$ & 86 & 081 & 39 \\
\hline & 20 & 4526 & 2833.7 & 85 & $06 \mathrm{I}$ & 36 \\
\hline & 2 I & $7 \quad 5320$ & $28 \quad 5.5$ & 8460 & 1043 & 34 \\
\hline & 22 & $8 \quad 1 \quad 17$ & 2735.1 & 8346 & 1027 & 32 \\
\hline & 23 & 916 & $27 \quad 2.4$ & 8228 & 1014 & 30 \\
\hline & 24 & 1718 & $26 \quad 27.4$ & 8106 & 1003 & 28 \\
\hline & 25 & $\begin{array}{lll}8 & 25 & 21\end{array}$ & +2649.9 & 97980 & 0.0994 & 10 27 \\
\hline
\end{tabular}

Die Elemente sind dieselben, die Iwanow für $1898 \mathrm{März} 25.0$ gefunden hat, nur dass die Jupiterstörungen erster Ordnung angebracht worden sind. Die Ephemeride wird hinreichend genau sein, um den Cometen aufzufinden, da die Correction derselben nicht 5:0 übersteigen wird. Eine weitere Ephemeride folgt.

$o^{\text {h }}$ M. Z. Berlin.

Kronstadt, Marine-Observatorium, I 90 I Juli 3 I.

Ch. Thonberg.

\section{Wiederauffindung des Encke'schen Cometen 1901 b.}

(Telegramm von Prof. Pickering in Cambridge, Mass.).

¿ncke's comet was observed by Wilson, Northfield, August 5.8924 Greenw. M. T., RA. app. $=6^{\mathrm{h}} 2^{\mathrm{m}} 2^{\mathrm{s}} .8$ PD. app. $=5^{8^{\circ}} 17^{\prime} 30^{\prime \prime}$. Pickering. «

Die Correction der Thonberg'schen Ephemeride beträgt hiernach $-1^{\mathrm{m}} 2^{\mathrm{s}}$ in RA. und $+1: 2$ in Decl. Kr.

\section{Mittheilung betreffend die Herausgabe eines neuen Cataloges der veränderlichen Sterne.}

Vom Vorstande der Astronomischen Gesellschaft ist die Herausgabe eines neuen Cataloges der veränderlichen Sterne beschlossen und die Ausführung dieser Arbeit der unterzeichneten Commission ubertragen worden. Die Commission erlaubt sich an die Beobachter der veränderlichen Sterne die Bitte zu richten, gröšsere, noch nicht publicirte Beobachtungsreihen, die zur Verbesserung der Elemente von Nutzen sein können, bald zu veröffentlichen oder Mittheilungen darïber. an das geschäftsführende Mitglied der Commission (Prof. G. Müller, Potsdam, Observatorium) gelangen zu lassen.

Zugleich macht die Commission bekannt, dass sie von jetzt an die definitive Benennung der neu entdeckten. Veränderlichen, sobald die Lichtänderung sicher festgestellt ist, ubernehmen wird. Eine Namensliste der in den letzten, Jahren aufgefundenen, bisher noch unbenannt gebliebenen Veränderlichen wird in der nächsten Zeit veroffentlicht werden.

Die Commission für die Herausgabe eines Cataloges der veränderlichen Sterne.

Duner, Hartwig, Miuller, Oudemans.

Inhalt zu Nr. 3732-33. G. Müller. Die Lichtcurve Algols in den Jahren 1878-188x. I77. - F. Scheiner. Ueber die Sternspectra mit theils hellen, theils dunklen Wasserstofflinien, 195. - A. A. Nijland. Das Mira-Maximum von August 1900. 199. - A. A. Nijland, S. L. Veenstra. Beobachtungen von Jupiterstrabanten. 203. - D. Gill. Observations of Comet r9or I. 203. Ch.Thonberg. Elemente und Aufsuchungs-Ephemeride des Encke'schen Cometen. 207. - Wiederauffindung des Encke'schen. Cometen 1901 b. 207. - Mittheilung betreffen'd die Herausgabe eines neuen Cataloges der veränderlichen Sterne. 207. 